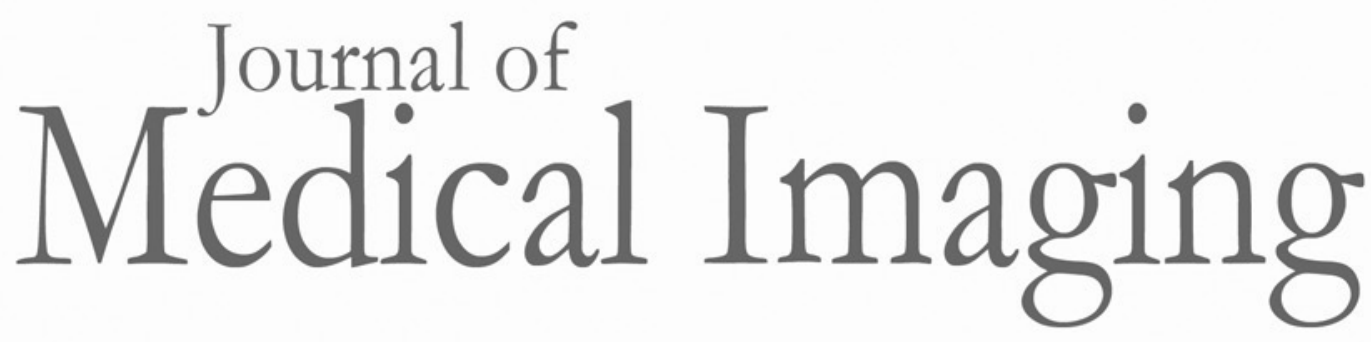

Medicallmaging.SPIEDigitalLibrary.org

\title{
Back-irradiated and dual-screen sandwich detector configurations for radiography (Erratum)
}

Anthony R. Lubinsky

Adrian Howansky

Hao Zheng

Wei Zhao 


\section{Back-irradiated and dual-screen sandwich detector configurations for radiography (Erratum)}

Anthony R. Lubinsky, Adrian Howansky, Hao Zheng, and Wei Zhao

Stony Brook University, Department of Radiology, Health Sciences Center, Stony Brook, New York, United States

[DOI: 10.1117/1.JMI.6.3.039801]

This article [J. Med. Imag. 6(3), 033501 (2019), doi: 10.1117/1.JMI.6.3.033501] was originally published online on 9 July 2019. In the original version of the article, there was a clerical error in the construction of the curves in Fig. 3(b) from a matrix of calculated results. This has no effect on the conclusions of the article. The original figure and the corrected figure are provided here (the caption did not change).

Original:
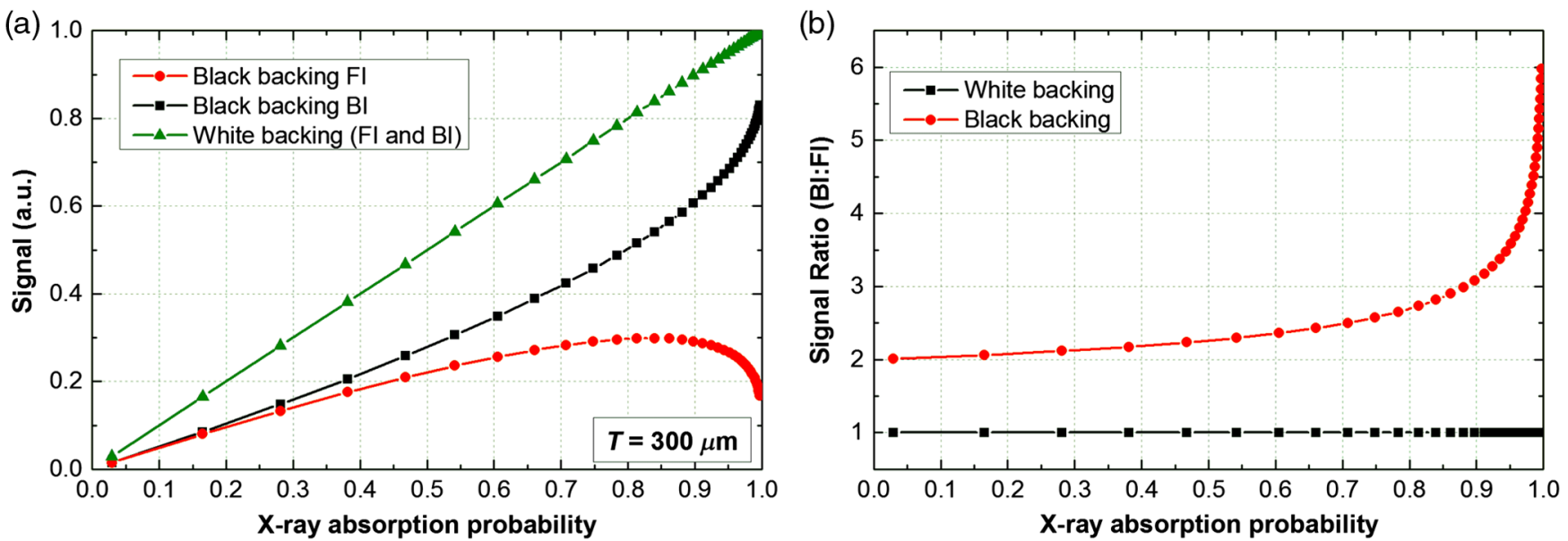

Fig. 3 Comparison of (a) DC signal for a single screen $(T=300 \mu \mathrm{m})$ with white or black backing, in FI and $\mathrm{BI}$, for a given exposure. (b) The corresponding ratio of $\mathrm{BI}: \mathrm{FI}$ signal.

Corrected:
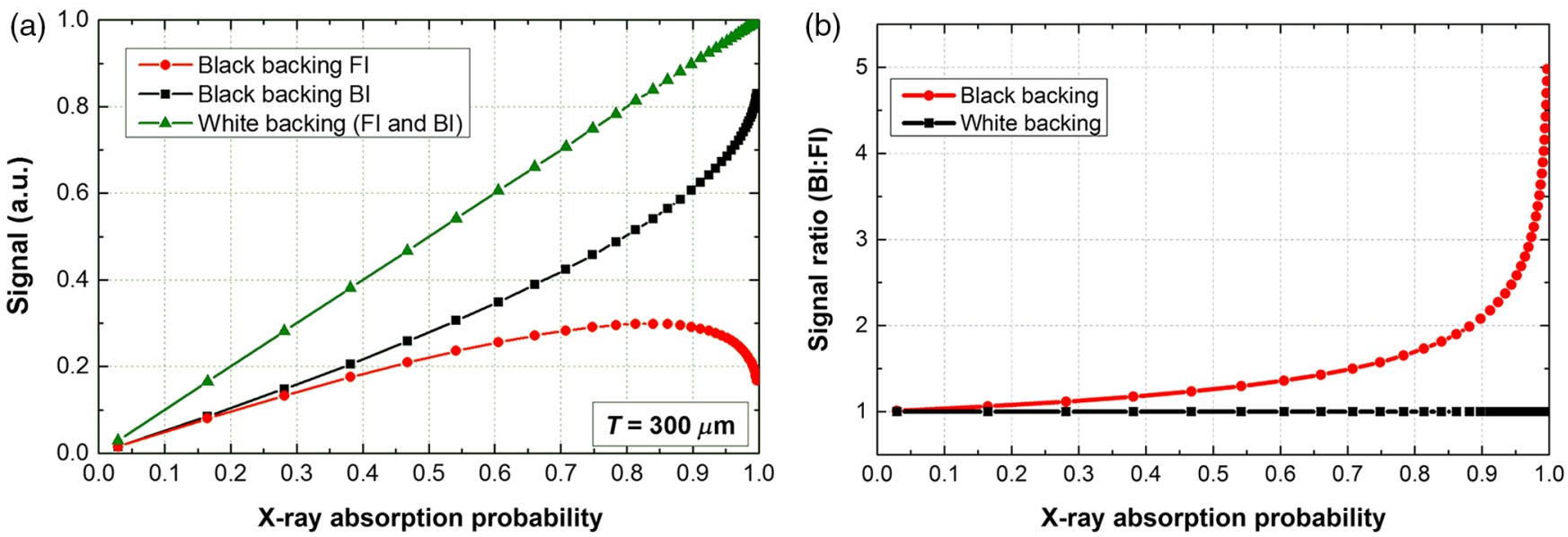

Fig. 3 Comparison of (a) DC signal for a single screen $(T=300 \mu \mathrm{m})$ with white or black backing, in FI and $\mathrm{BI}$, for a given exposure. (b) The corresponding ratio of $\mathrm{BI}: \mathrm{Fl}$ signal.

This article was corrected online on 25 July 2019. It appears correctly in print. 